\title{
New Measurement Method in Drug Sensing by Direct Total-Charge Detection in Voltammetry
}

\author{
Simone Aiassa*†, José David González Martínez ${ }^{\dagger}$, Danilo Demarchi* ${ }^{* \dagger}$, and Sandro Carrara ${ }^{\dagger}$ \\ ${ }^{*}$ Department of Electronics and Telecommunications, Politecnico di Torino, Turin, Italy \\ ${ }^{\dagger}$ Integrated Systems Laboratory, École Polytechnique Fédérale de Lausanne, Lausanne, Switzerland \\ Email: simone.aiassa@polito.it
}

\begin{abstract}
Electrochemical biosensors are promoting point-ofcare and wearable instrumentation due to their high versatility in measuring human metabolites. There is a considerable number of biological compounds that can be detected and measured through voltammetry based techniques. Voltmmetry some times requires peak identification and quantification that are non-trivial to be efficiently implemented by automatic instrumentation. To overcome the complexity of automatic peak estimation, we propose here an instrumentation circuit for edge-computing in pharmacology relying on an entirely novel measurement method via TotalCharge Detection in Cyclic voltammetry (TCDC). Namely, our TCDC method innovatively applies the coulometry measurement to the well-established voltammetry procedure. The proposed instrumentation accumulates the total charge exchanged in the faradaic process, exploiting a Nagaraj integrator as charge suppressor to fit the application-specific constraints. The work shows accurate simulations of the TCDC circuit on a set of experimental measures, acquired on paracetamol as benchmark drug. The proposed measurement technique and the developed circuit are compared to the peak detection method usually adopted in literature. The results demonstrate that the proposed system is a perfect trade-off between the doubled limit-of-detection and a tenfold reduction in measurement errors. At the same time, we eliminate any need for data oversampling and processing, promoting the TCDC as an efficient new measurement method for point-of-care and wearable monitoring of biological compounds.
\end{abstract}

Index Terms-Biosensor, Measurement Method, Point-of-Care, TCDC, Voltammetry Based Sensing

\section{INTRODUCTION}

Patient's real-time monitoring of drug response could improve the effectiveness of medical treatments, helping to avoid over or under dosage during therapy [1]. Several methods have been proposed for detecting biological compounds in human tissue. In the pharmaceutical analysis, electrochemical techniques are widely adopted, being precise, low cost, and suitable to selectively identify a large number of compounds [2]. In particular, Voltammetry Based Sensing (VBS) techniques, like Cyclic Voltammetry (CV) and Differential Pulse Voltammetry (DPV), are amperometric sensing methods widely adopted to determine the concentration of several drugs [2], [3]. CV and DPV are required to compensate for poor stability and high variability over time of reference electrodes and reference potentials. While VBS methods exploit on-line monitoring of patients [4], [5], most of the pointof-care or wearable platforms present in literature usually calculate the drug concentration offline, instead of leveraging on VBS techniques for direct determination of concentration. Indeed, in point-of-care and wearable system, the difficulty of automatically retrieve the final information directly by the electronics limits the application of VBS.

Typically, the VBS system consists of a sensor to measure the biological compound and an electronic unit (potentiostat) to drive and read the sensor. The sensor is typically a three electrodes electrochemical cell, composed by a Reference Electrode (RE), a Counter Electrode (CE) and a Working Electrode (WE) [6]. In order to perform a CV, the potentiostat drives a voltage ramp within a specific window depending on the target biological compound and then the potentiostat samples the current produced by the electrochemical reaction induced in the cell. Based on the shape of the so-called voltammogram (current upon the voltage), it is possible to extract information, e.g. the concentration of the drug present in the sample. One of the best features which help for the discrimination of drugs is the Redox/Oxidation current peak produced by the faradaic process [7]. To reliably detect those peaks and correctly quantify the related drugs is usually required to implement a custom digital architecture [8], adopt an operating system based processor [9], or process the information offline [10]. Unfortunately, all of those techniques require power and area demanding electronic architectures, with suitable sampling rate [11]. Those factors conflict with the edge-computing paradigm [12] needed for the connected point-of-care or wearable monitoring.

We present here a novel measurement method based on Total-Charge Detection in Cyclic-voltammetry (TCDC) instead of the full-voltammogram analysis. This approach scales down significantly the complexity at the edge-computing in drugs detection systems. The TCDC originally applies a charge measurement on the top of a standard voltammetry-based procedure to detect, through coulometry, the concentration of the target biological compound. The paper also shows a complete circuit implementation of our TCDC method to prove that it is feasible to implement it in consequent medical instrumentations. The proposed instrumentation and the new TCDC method are both validated using real data. Acquisitions with electrochemical sensors on paracetamol ( $\mathrm{N}$-acetil-paraamminofenolo) are here considered as the benchmark of electrochemical determination of pharmacological drugs [13]. 


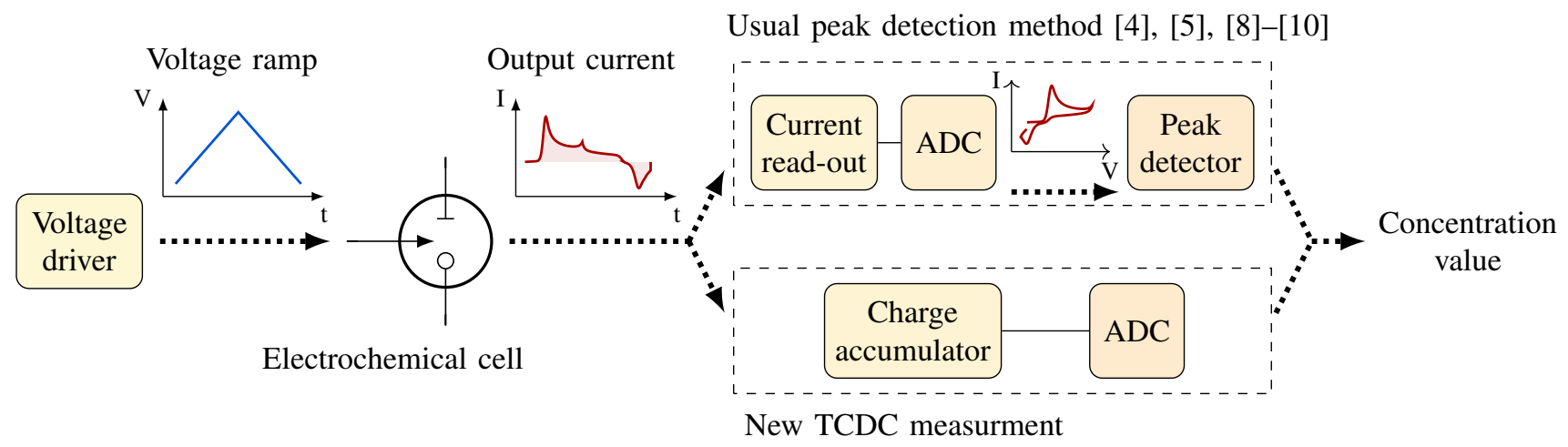

Fig. 1: In the TCDC measurement method, the total charge is acquired to determine the drug concentration, avoiding computation and oversampling typically needed by the usual peak detection method .

\section{TCDC MeAsurement Method}

Amperometric electrochemical detection methods usually rely on the determination of compounds by measuring the faradaic current. Differently, the TCDC measurement method presented here focuses on the total-charge exchanged in the electrochemical cell due to the faradic process. The coulometry method (the measurement of the charge) has already been applied for the determination of biological compounds [14]. However, to the best of our knowledge, the measurement of charge has never been applied in VBS method for continuous drug monitoring, since standard current sampling in $\mathrm{CV}$ is usually exploied [15]. Several electro-active therapeutic drugs can be involved in an electrochemical reaction [2], in which the total net charge exchanged $Q_{t o t}$ is defined as the sum of the faradaic process $Q_{F}$ and the non-faradaic one $Q_{N F}$, as described in (1).

$$
Q_{t o t}=Q_{F}+Q_{N F}
$$

If we approximate faradaic charge with the Faraday's laws of electrolysis and the non-faradaic charge to the double-layer capacitance effect on the interface [6], then the total charge at the electrochemical interface is:

$$
Q_{t o t}=n F N+C_{d} V
$$

Where $n$ is the number of electrons transferred in the reaction, $F$ the Faraday constant, $N$ the number of moles involved in the reaction, $C_{d}$ the equivalent capacitance of the double layer, and $V$ the potential applied at the interface. From (2) it is possible to derive the calibration equation:

$$
Q_{t o t}=Q_{0}+S \cdot C
$$

which relates $Q_{t o t}$ and the concentration of target analyte $C$. $S$ is the Sensitivity defined as Coulomb per Molar (C/M) and $Q_{0}$ is the total non-faradaic exchanged charge. To a first approximation, $Q_{0}$ does not depend on the analyte concentration, and it can be extracted as a calibration constant.

Fig. 1 schematically explains the proposed TCDC measurement in comparison to the usual peak detection method presented in literature [4], [5], [8]-[10]. Both in the standard
$\mathrm{CV}$ procedure and TCDC, a voltage ramp is applied to the electrochemical cell. In CV, the system samples the output current, although, in TCDC, the instrumentation accumulates the charge to measure the total net charge exchanged during the whole electrochemical process. Therefore, the TCDC does not require any more oversampling of the current to extract a feature from its shape. The here proposed measurement system gives the concentration value directly in the form of a voltage linearly proportional to the target drug concentration. In terms of instrumentation, TCDC presents a drastic reduction in complexity, eliminating both processing and oversampling required in $\mathrm{CV}$ techniques, paving the road for several applications where standard microcontroller-based systems are not suitable.

\section{IMPLEMENTATION}

To implement the TCDC method, a charge to voltage converter is designed to accumulate the total charge produced in the electrochemical process. The output of the converter is implemented to input an ADC to get a digital value linearly proportional to the input concentration. The design of the circuit is constraint by the time involving the electrochemical reaction. For example, the time required to perform a CVdetection on paracetamol is around $10 \mathrm{~s}$ at a Scan Rate (SR) of $0.2 \mathrm{~V} / \mathrm{s}$ [4], [5], [11]. Even though a simple integrator can perform the charge-to-voltage conversion, a standard inverting integrator is not suitable for the TCDC since the long-time constant will requires area-demanding component, usually avoided in any conventional CMOS implementation. In order to reduce the capacitor sizes, attenuation techniques are applied, helping to eliminate part of the input charge [16]. In particular, the parasitic-insensitive Nagaraj integrator [17] exploits the attenuation.

\section{A. Circuit Implementation}

In order to demonstrate the principle of the TCDC method, the here proposed instrumentation is fully-based on Switch Capacitors (SC), without resistance components, planning a future CMOS implementation. Fig 2 shows the implemented TCDC instrumentation: in the first stage, $\mathrm{A}_{1}$ operates as Nagaraj charge-attenuator, while in the second stage, $\mathrm{A}_{2}$ operates 


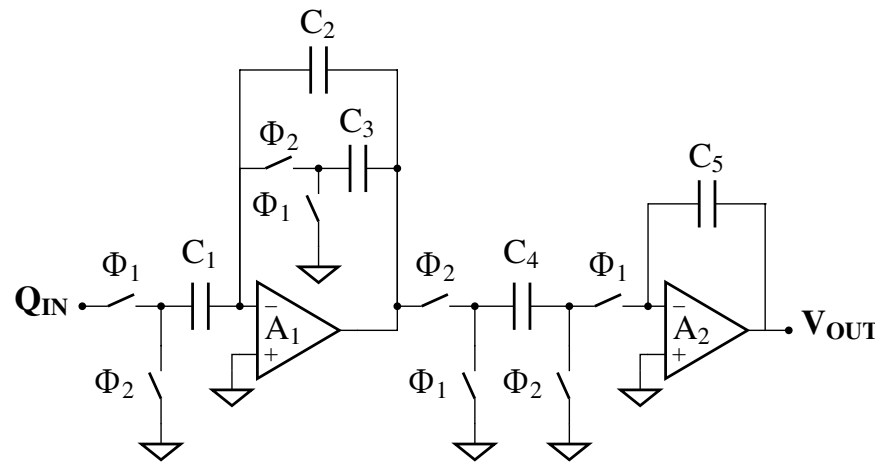

Fig. 2: TCDC instrumentation. The Nagaraj integrator $\left(A_{1}\right)$ and the SC integrator $\left(\mathrm{A}_{2}\right)$ accumulate and convert the total input charge into the output voltage, during the two nonoverlapped clock phase $\left(\Phi_{1}, \Phi_{2}\right)$

as standard SC integrator. Due to the long time period of charge accumulation required by the electrochemical drug detection, the Nagaraj topology does not operate as an ideal integrator, since the long time constant suppresses its normal operating behaviour. Therefore, the proposed instrumentation relies on a two-stage circuit with the novelty of taking advantages by [17] to attenuate the input charge, and to achieve the specific purpose goal. The SCs are controlled by a two not-overlapped phase of the same clock $\left(\Phi_{1}\right.$ and $\left.\Phi_{2}\right)$. During $\Phi_{1}$, the charge is transferred from $C_{1}$ to $C_{2}$, and sampled by $\mathrm{C}_{3}$. In $\Phi_{2}, \mathrm{C}_{1}$ withdraws the charge stored in $\mathrm{C}_{2}$, while $\mathrm{C}_{1}$ redistributes its charge with $\mathrm{C}_{2}$.

The system is designed to fit a clock frequency of $32.768 \mathrm{kHz}$, the most widely adopted resonator in low-cost systems [18] and the supply voltage is set to $1.8 \mathrm{~V}$ matching the CMOS $180 \mathrm{~nm}$ tecnology for future developments. Considering the first stage, $C_{2}$ is equal to $20 \mathrm{fF}$, while $\mathrm{C}_{1}$ and $\mathrm{C}_{3}$ are $70 \mathrm{aF}$, value slightly above the current size limit of CMOS $180 \mathrm{~nm}$ technology [19], minimizing therefore the size of the circuit until the technological limit. In the second stage, $\mathrm{C}_{4}$ is $3 \mathrm{fF}$ and $\mathrm{C}_{5}$ is $40 \mathrm{pF}$ to have an equivalent resistance of $10 \mathrm{G} \Omega$ to separate the two stages and avoid leakages. Foldedcascode Operational Transconductance Amplifiers (OTA) implement both operational amplifiers in the design. All the values mentioned above are selected by a process variation analysis. During the simulation, tolerance values obtained by literature [19] are considered to cover extreme cases and to guarantee the compensation of process variation by clock frequency tuning. Particularly, this analysis is performed on the value of $\mathrm{C}_{1}$ and $\mathrm{C}_{3}$ due to their small value. Supposing a typical process variation of $50 \%$, both capacitor values are tested in the range $70 \pm 30 \mathrm{aF}$.

\section{B. Circuit Behaviour}

Fig. 3 presents the behaviour of the proposed TCDC instrumentation. For the sake of simplicity, the current produced by the sensor (top left) represents the input charge. The Nagaraj integrator suppresses partially the input charge producing an
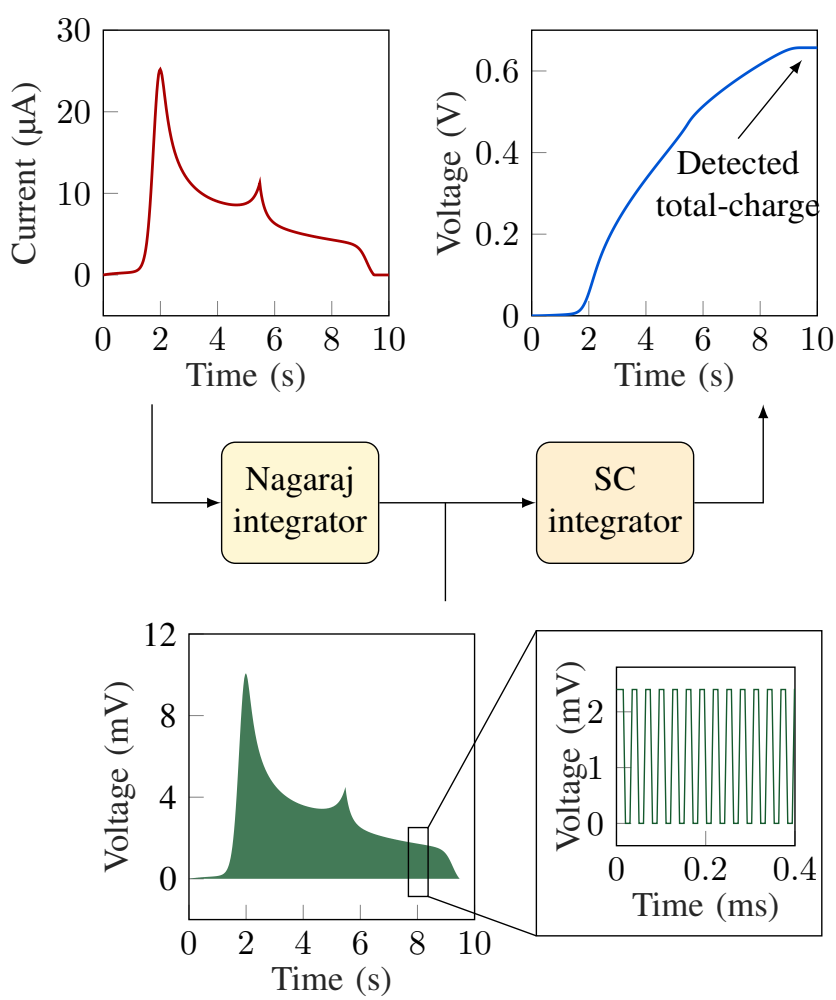

Fig. 3: TCDC circuit behaviour. The Nagaraj integrator suppresses partially the input charge while the SC integrator output-voltage is proportional to the total charge.

attenuated switching voltage signal (bottom). The SC integrator accumulates the charge from the begin of the $\mathrm{CV}$ procedure until its end. The maximum voltage reached by the output can be held, sampled, and fed to an ADC as the final detected value of the total charge. The voltage fits the conversion range of the $\mathrm{ADC}$ avoiding saturation and can be adapted to a wide range of measure tuning either statically by the component size or dynamically by the clock frequency.

\section{VALIDATION}

Real data sets are collected experimentally in a lab environment to validate the TCDC method and its related instrumentation. The data are used for both analytical comparisons as well as input for simulating the implemented measurement circuit.

\section{A. Materials and Method}

Paracetamol is the drug selected as benchmark, and its powder is dissolved in Phosphate Buffer Saline (PBS) solution at $\mathrm{pH} 7.4$, which acts as background electrolyte. All the chemicals are from Sigma Aldrich ${ }^{\circledR}$. A Screen-Printed Electrode (SPE), namely DropSens DRP-110 composed of a carbon-based WE (4 mm of diameter), a carbon $\mathrm{CE}$, and a silver RE, is immersed in the solution to detect the drug via the electrochemical reaction. The sensor is connected to the Metrohm Autolab PGSTAT 302N, driven by the software Nova 1.11 , which is a lab measurement instrumentation designed 
to perform high-quality and high-resolution $\mathrm{CV}$ procedures. Subsequent concentration steps of $50 \mu \mathrm{M}$ of paracetamol in the pharmacological therapeutic range of the target drug $(50: 300 \mu \mathrm{M})$ are added to the buffer solution. The instrumentation performs each time a full $\mathrm{CV}$ voltammogram at a Scan Rate (SR) of $0.2 \mathrm{~V} / \mathrm{s}$ in the voltage range between $-0.1 \mathrm{~V}$ and $1.1 \mathrm{~V}$. In order to consider the experimental variability, each measure is repeated three times with a new electrode. The lab instrument samples the current produced by the sensor at a rate of around 33.3 sample/s (400 samples per CV) and stored.

\section{B. Simulation and Data Analysis}

In the simulation of the implemented circuit, the waveforms obtained by the lab measure are applied as input vectors. The same waveforms are also analytically processed to have a fair comparison with the usual offline methods of peak estimation. The analytical processing of the data is performed in Matlab ${ }^{\circledR}$, the built-in function findpeaks returns the height of the oxidation peak shown by the voltammogram. Meanwhile, the built-in function trapz mathematically estimates the totalcharge considering the trapezoidal numerical integration. The electrical simulations are performed with OrCAD ${ }^{\circledR}$ PSpice $^{\circledR}$, and the output voltage is sampled after $12 \mathrm{~s}$, namely, at the end of the CV exitation.

\section{RESULTS}

The data collected through the experiment are here analysed to define better the capability and limitation of the proposed TCDC method. The analysis also helps to understand the performance related to different possible range of charge accumulation in the CV. After the simulation, the detecting performances of the proposed measurement circuit are compared with the analytical results obtained with the conventional peak detection method.

\section{A. Measurement Results}

Fig. 4 presents the voltammogram curves acquired by the lab instrument and the SPE electrode on the prepared paracetamol samples. The increasing of paracetamol concentration reflects on a linear increase of both oxidation and reduction peaks.

According to the definition of TCDC provided in Section II, the total charge exchanged in the faradaic process expresses the concentration of the analyte. Fig. 5 presents the current versus time acquired during the lab test at a concentration of $300 \mu \mathrm{M}$. Considering a standard $\mathrm{CV}$ is possible to define different intervals of integration in time, which are related to different ranges of charge accumulation. The range $\mathrm{A}$ is related to the first part of the voltammogram, where the positive-increasing voltage drives the electrochemical cell. The range $\mathrm{A}+\mathrm{B}$ consider the total positive-only charge in all the voltammogram. Finally, the negative charges highlighted in $\mathrm{C}$ can be both considered as discharge, obtaining the total natural charge in $\mathrm{CV}$ (range $\mathrm{A}+\mathrm{B}-\mathrm{C}$ ) or, adding a rectifier, as another additive charge, obtaining the cumulative total charge (interval

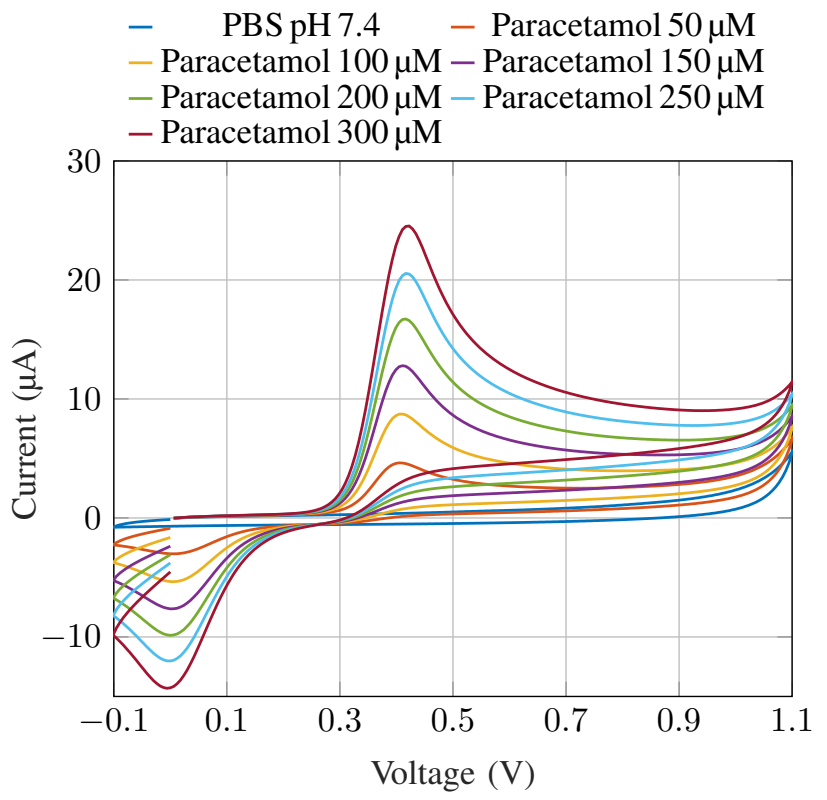

Fig. 4: Collected CV waveform during lab experiment, considering different concentration of paracetamol in the therapeutic range.

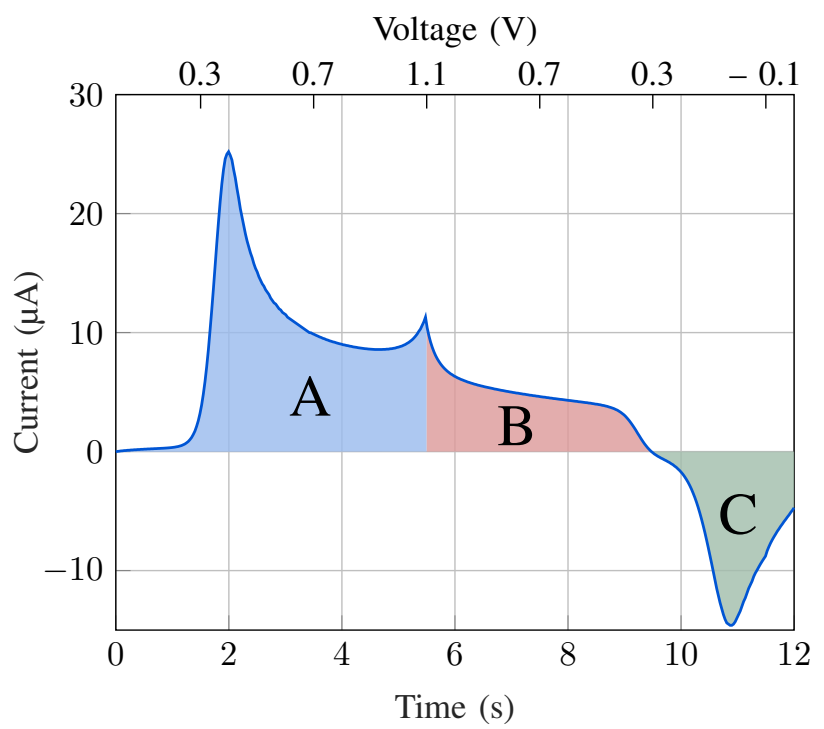

Fig. 5: Possible range of charge accumulation in applying TCDC. Positive increasing only excitation waveform (A), positive only charge $(A+B)$, natural total charge $(A+B-C)$, or cumulative total charge $(A+B+C)$.

$\mathrm{A}+\mathrm{B}+\mathrm{C})$. Quite simple instrumentations can implement all the interval.

Table I compares the possible interval of charge accumulation considering the sensitivity (as Coulumb per Molar, $S$, in the equation (3)), offset ( $Q_{0}$ in (3)), linearity, and Limit-ofDetection (LOD). The sensitivity increases enlarging the range of integration, presenting its maximum in the interval $A+B+C$. The regression coefficient of the obtained calibration curve 
TABLE I: Comparison between possible range of charge accumulation in TCDC measurement method.

\begin{tabular}{lcccc}
\hline & $\mathrm{A}$ & $\mathrm{A}+\mathrm{B}$ & $\mathrm{A}+\mathrm{B}-\mathrm{C}$ & $\mathrm{A}+\mathrm{B}+\mathrm{C}$ \\
\hline Description & $\begin{array}{c}\text { Increasing positive } \\
\text { only CV }\end{array}$ & $\begin{array}{c}\text { Positive-only charge } \\
\text { Sensitivity }(\mathrm{nC} / \mu \mathrm{M})\end{array}$ & $\begin{array}{c}\text { Natural total charge } \\
\text { Offset }(\mu \mathrm{C})\end{array}$ & $\begin{array}{c}\text { Cumulative total } \\
\text { charge }\end{array}$ \\
Linearity $\left(\mathrm{r}^{2}\right)$ & $5.27 \pm 1.52$ & $5.59 \pm 2.57$ & $3.92 \pm 2.45$ & $260.7 \pm 6.8$ \\
LOD $(\mu \mathrm{M})$ & .99997 & .99997 & .99997 & $.26 \pm 2.70$ \\
\hline
\end{tabular}

evaluates the linearity, and the increase in the accumulation time does not affect this parameter. The LOD represents the minimum concentration of drug the system can detect and, in this work, the LOD is evaluated as three times the standard deviation of the measure, divided by the sensitivity. The results demonstrate that the application of the TCDC at the interval $\mathrm{A}+\mathrm{B}-\mathrm{C}$, which is the total charge exchanged during the $\mathrm{CV}$, presents a considerable reduction of performance showing a $69 \%$ increase in the LOD. Meanwhile, the interval A+B, which is the accumulation of positive-only flowing charge, presents the best performance. For this reason, all simulations and the following considerations are then conducted considering the positive-only charge. Moreover, this choice is fully compatible with the circuit implemented and described in Section III.

\section{B. Simulation Results}

Fig. 6 displays a comparison in the calibration curves obtained by both analytical-processing and simulation. Namely, Fig. 6a shows the resulting calibration obtained by measuring the paracetamol concentration with the extraction of the current peak. In contrast, Fig. 6b displays the analytical computation of the total-charge as integral of the current flowing in the electrochemical cell. Instead, Fig. 6c presents the calibration curve obtained by performing the TCDC circuit simulation. As visible in Fig. 6, all the method are suitable to calibrate linearly with the sensor data.

Table II compares the detection performance in term of sensitivity, offset, linearity, and LOD. The sensitivity can not be compared in absolute terms due to different outputs as obtained by the same transducer, and the same is valid for the offset. On the other hand, the linearity is the first indicator of the performance of the TCDC instrumentation since it presents an increase from 0.99970 up to 0.99994 , with respect to the peak detection method. Applying the TCDC method, the LOD increases due to the trade-off between the proposed dramatic reduction in complexity and the quality of the measure. The LOD increases because TCDC collects all the charge in the $\mathrm{CV}$, therefore, acquiring a higher background noise related to the not-faradaic phenomena [6]. Although, we here demonstrate that the increase of LOD can be kept under control since it is limited approximately to 2.2 times with respect to peak detection by conventional methods. The LOD always remains one order of magnitude lower than the typical minimum

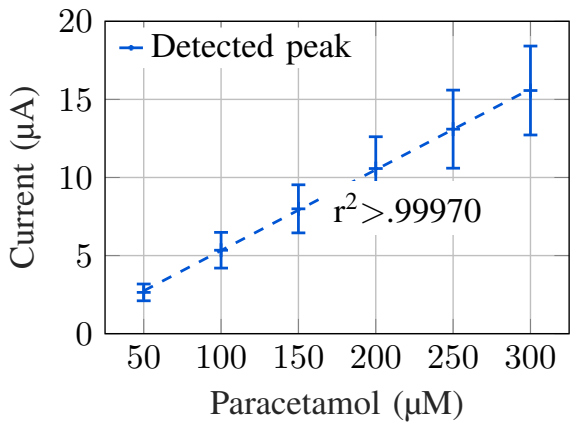

(a)

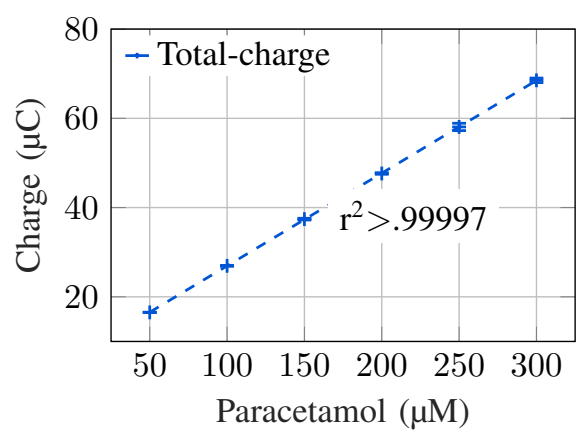

(b)

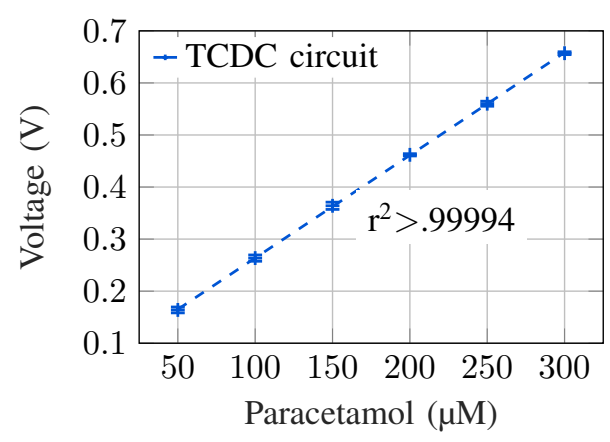

(c)

Fig. 6: Calibration curves obtained by extractionon of current peak (a), analytical total charge (b), and TCDC circuit simulation (c). 
TABLE II: Comparison between peak detection method and TCDC, both analytical and simulation results.

\begin{tabular}{lccc}
\hline & $\begin{array}{c}\text { Peak } \\
\text { detection* }\end{array}$ & $\begin{array}{c}\text { Total } \\
\text { charge* }\end{array}$ & $\begin{array}{c}\text { TCDC } \\
\text { circuit }\end{array}$ \\
\hline Sensitivity & $\begin{array}{c}51.7 \pm 9.2 \\
\mathrm{nA} / \mu \mathrm{M}\end{array}$ & $\begin{array}{c}207.8 \pm 2.8 \\
\mathrm{nC} / \mu \mathrm{M}\end{array}$ & $\begin{array}{c}1.97 \pm 0.03 \\
\mathrm{mV} / \mu \mathrm{M}\end{array}$ \\
Offset & $0.15 \pm 0.15$ & $5.59 \pm 2.57$ & $66.4 \pm 9.0$ \\
Linearity $\left(\mathrm{r}^{2}\right)$ & 0.99970 & 0.99997 & 0.99994 \\
Limit of & $1.93 \pm 0.94$ & $5.30 \pm 0.09$ & $6.09 \pm 0.12$ \\
detection & $\mu \mathrm{M}$ & $\mu \mathrm{M}$ & $\mu \mathrm{M}$ \\
\hline
\end{tabular}

* Matlab $^{\circledR}$ analytical results.

pharmacological concentration. Therefore, the quality of the measurements is not compromised. Moreover, we prove here that the proposed method significantly reduces the error on the single drug measure (defined as a statistical error, that is three times the standard deviation), clearly visible directly in Fig. 6. Namely, our approach scales the measurement error down from $17.8 \%$ to just $1.7 \%$ because of the higher obtained sensitivity which reflects on a higher resolution.

\section{CONCLUSION}

We have introduced a novel measurement method, we called TCDC (Total Charge Detection in Cyclic Voltammetry), for drugs detection. We successfully demonstrated that the related innovative instrumentation provides better calibration (higher linearity and tenfold measurement error reduction), thanks to an edge-computing lower-complexity method which removes both processing and oversampling, trading off a slightly more than doubled LOD. Hence, the proposed method fits the requirement for point-of-care and wearable real-time monitoring. Future works will include the implementation in CMOS $180 \mathrm{~nm}$ technology, already considered by design, and the validation of different pharmacological compounds.

\section{ACKNOWLEDGMENT}

This work is supported by Politecnico di Torino and Compagnia di San Paolo under the initiative "Joint research projects with top universities".

\section{REFERENCES}

[1] T. Buclin, V. Gotta, A. Fuchs, N. Widmer, and J. Aronson, "An agenda for UK clinical pharmacology: Monitoring drug therapy," British Journal of Clinical Pharmacology, vol. 73, no. 6, pp. 917-923, 2012.
[2] G. Ozcelikay, L. Karadurmus, S. I. Kaya, N. K. Bakirhan, and S. A. Ozkan, "A review: New trends in electrode systems for sensitive drug and biomolecule analysis," Critical Reviews in Analytical Chemistry, pp. 1-14, 2019.

[3] C. Baj-Rossi, G. De Micheli, and S. Carrara, "Electrochemical detection of anti-breast-cancer agents in human serum by cytochrome p450-coated carbon nanotubes," Sensors, vol. 12, no. 5, pp. 6520-6537, 2012.

[4] F. Stradolini, A. Tuoheti, P. Motto Ros, D. Demarchi, and S. Carrara, "Raspberry Pi based system for portable and simultaneous monitoring of anesthetics and therapeutic compounds," in 2017 New Generation CAS (NGCAS), pp. 101-104, Genova, Italy, 2017.

[5] S. Aiassa, F. Stradolini, A. Tuoheti, S. Carrara, and D. Demarchi, "Quasi-digital biosensor-interface for a portable pen to monitor anaesthetics delivery," in 2019 15th Conference on Ph.D Research in Microelectronics and Electronics (PRIME), pp. 265-268, Lausanne, Switzerland, 2019.

[6] A. J. Bard, L. R. Faulkner, J. Leddy, and C. G. Zoski, "Potential sweep method," in Electrochemical Methods: Fundamentals and Applications, pp. 226-260, New York: Wiley, 2 ed., 1980.

[7] S. Carrara, Bio/CMOS Interfaces and Co-Design. New York: SpringerVerlag, 1 ed., 2013.

[8] M. Schirmer, F. Stradolini, S. Carrara, and E. Chicca, "FPGA-based approach for automatic peak detection in cyclic voltammetry," in 2016 IEEE International Conference on Electronics, Circuits and Systems (ICECS), (Monte Carlo, Monaco), pp. 65-68, 2016.

[9] P. Bezuidenhout, S. Smith, K. Land, and T. . Joubert, "A low-cost potentiostat for point-of-need diagnostics," in 2017 IEEE AFRICON, (Cape Town, South Africa), pp. 83-87, 2017.

[10] I. Ny Hanitra, F. Criscuolo, S. Carrara, and G. De Micheli, "Multi-target electrolyte sensing front-end for wearable physical monitoring," in 2019 15th Conference on Ph.D Research in Microelectronics and Electronics (PRIME), pp. 249-252, Lausanne, Switzerland, 2019.

[11] S. Aiassa, S. Carrara, and D. Demarchi, "Optimized sampling rate for voltammetry-based electrochemical sensing in wearable and IoT applications," IEEE Sensors Letters, vol. 3, no. 6, pp. 1-4, 2019.

[12] M. Capra, R. Peloso, G. Masera, M. Ruo Roch, and M. Martina, "Edge computing: A survey on the hardware requirements in the internet of things world," Future Internet, vol. 11, no. 4, p. 100, 2019.

[13] E. Roberts, V. D. Nunes, S. Buckner, S. Latchem, M. Constanti, P. Miller, M. Doherty, W. Zhang, F. Birrell, M. Porcheret, et al., "Paracetamol: not as safe as we thought? A systematic literature review of observational studies," Annals of the Rheumatic Diseases, vol. 75, no. 3, pp. 552-559, 2016.

[14] I. Anshori and H. Suzuki, "Electrochemical microdevices for proteins based on coulometry coupled with silver metallization," in 2017 19th International Conference on Solid-State Sensors, Actuators and Microsystems (TRANSDUCERS), (Kaohsiung, Taiwan), pp. 1555-1558, 2017.

[15] C. Baj-Rossi, T. R. Jost, A. Cavallini, F. Grassi, G. De Micheli, and S. Carrara, "Continuous monitoring of naproxen by a cytochrome p450based electrochemical sensor," Biosensors and Bioelectronics, vol. 53, pp. 283-287, 2014.

[16] S. Tumati, Design of large time constant switched-capacitor filters for biomedical applications. PhD thesis, Texas A\&M University, 2005.

[17] K. Nagaraj, "A parasitic-insensitive area-efficient approach to realizing very large time constants in switched-capacitor circuits," IEEE Transactions on Circuits and Systems, vol. 36, no. 9, pp. 1210-1216, 1989.

[18] H. G. Barrow, T. L. Naing, R. A. Schneider, T. O. Rocheleau, V. Yeh, Z. Ren, and C. T. Nguyen, "A real-time $32.768-\mathrm{kHz}$ clock oscillator using a $0.0154-\mathrm{mm}^{2}$ micromechanical resonator frequency-setting element," in 2012 IEEE International Frequency Control Symposium Proceedings, (Baltimore, MD, USA), pp. 1-6, 2012.

[19] A. Hastings, The Art of Analog Layout, vol. 2. New Jersey: Pearson Prentice Hall, 2006. 